\title{
Salmonella enterica serovar Typhimurium typing by prophage-specific PCR
}

\begin{abstract}
Correspondence
Ivan Rychlik

rychlik@vri.cz
\end{abstract}

Received 16 November 2007

Revised 15 December 2007

Accepted 22 February 2008
Ivan Rychlík, Helena Hradecka and Marcela Malcova

Veterinary Research Institute, Hudcova 70, 62100 Brno, Czech Republic

\section{INTRODUCTION}

Gastrointestinal disorders in humans are frequently caused by Salmonella enterica subsp. enterica. Although more than 2000 different serovars can be differentiated in Salmonella enterica subsp. enterica, serovars Enteritidis and Typhimurium ( $S$. Typhimurium) are responsible for the vast majority of human salmonelloses (Galanis et al., 2006). To differentiate Salmonella strains originating from outbreaks, phage typing, antibiotic-resistance typing, plasmid-profile analysis and PFGE are the most frequently used methods. Out of the DNA-based typing methods, PGFE is the method of choice for Salmonella, and also for other bacterial species, despite the fact that the reasons for the variability in the size of the restriction fragments are not known.

The genome of $S$. Typhimurium strain LT2 has been known since 2001 (McClelland et al., 2001), and this has allowed in silico identification of restriction sites, and prediction of PFGE profiles. Although not annotated in GenBank, two additional $S$. Typhimurium genomes, specifically those of SL1344 and the pentadrug-resistant DT104 strain, are publicly available on the website of the institution that performed the sequencing. When we searched for the $\mathrm{Xba \textrm {I }}$ sites in the sequenced genomes to verify the PFGE profiles (Van Immerseel et al., 2004; Hradecka et al., 2006; Matiasovicova et al., 2007), we observed that the XbaI sites frequently overlapped with the prophage sequences. In other cases, the presence of a prophage was shown to have led to an increase in the size of DNA fragments generated by $\mathrm{XbaI}$ restriction endonuclease. When we realized this, we assumed that a rational design of PCRs targeted to various mobile

PCR primer sequences and detailed PCR data are available with the online version of this paper.
DNA sequences, mostly of prophage origin, might be a very sensitive tool for differentiation of $S$. Typhimurium. Also, since the positive and negative results of individual PCRs can be easily shared among laboratories, such a typing system might be simple to perform worldwide. In this study, we showed that this assumption was correct, and that a combination of four triplex PCRs enabled differentiation of $S$. Typhimurium field strains, with essentially the same sensitivity as their differentiation by PFGE.

\section{METHODS}

Bacterial strains and culture conditions. In the first part of the study, we used 58 field strains that originated from different hosts; these were isolated in the Czech Republic between 2004 and 2007. This set of strains was used for typing by individual PCRs specific for 22 different target sequences. After this round of typing, the results obtained were evaluated, and the number of targets selected for PCR typing was reduced to 12 . New primer pairs were designed to allow use of these PCRs in four triplex PCRs (see below). Strains listed in Table 1 were characterized by the triplex PCRs and PFGE for comparison. In addition, the original host of each strain was known (except for two control laboratory strains, LT2 and SL1344), and the phage type according to Anderson et al. (1977) was available (except for 13 strains, three of which were control laboratory strains LT2, SL1344 and F98).

Sequence data analysis. The genomic sequence of $S$. Typhimurium LT2 strain is available (GenBank accession no. NC_003197). Raw sequences of S. Typhimurium SL1344 and S. Typhimurium DT104 were downloaded from http://www.sanger.ac.uk/Projects/Salmonella/, and then mutually aligned. In this way, sequences present in one of the genomes, but absent in at least one of the remaining two strains, were identified. In another analysis, $X b a I$ sites were identified in the genomes of all three sequenced strains, and superimposed over a map of predicted variable DNA sequences. 
Table 1. S. Typhimurium field strains used in this study

\begin{tabular}{|lrl|}
\hline Phage type & No. of strains & \\
\hline DT1 & 3 & Poultry (2), wild boar (1) \\
DT2 & 10 & Pigeon (9), duck (1) \\
DT12 & 1 & Human (1) \\
DT14 & 1 & Cattle (1) \\
DT104 & 62 & Human (18), pig (17), cattle (14), poultry (6), cat (2), environment (2), pigeon (2), food (1) \\
DT114 & 1 & Human (1) \\
DT120 & 3 & Human (1), pig (1), cattle (1) \\
DT166 & 1 & Pigeon (1) \\
DT193 & 1 & Cattle (1) \\
DT195 & 1 & Pigeon (1) \\
U & 2 & Human (1), parrot (1) \\
RDNC & 3 & Cattle (2), pheasant (1) \\
Not known & 13 & Cattle (5), pig (3), unknown (2), poultry (2), human (1) \\
\hline
\end{tabular}

*Values in parentheses indicate the number of strains originating from a particular host.

PCR design. Based on the identification of variable DNA sequences in the genomes of three strains, and data originating from microarray genomotyping (Porwollik et al., 2004; Reen et al., 2005; Matiasovicova et al., 2007), 22 individual PCRs specific for the potentially variable DNA sequences were designed. These included the STM05170529 deletion, the left junction of SGI1, and STM908, STM2619, STM2697, STM2705, gipA, artA, sodCI, bim2, eac, sieB, g8, gtrA, hldD, $s o p E$, Fels Sop, $g p 36, \operatorname{rrt} T$, spvB, oafA and $f l j B$ (for primer sequences and the results of these PCRs, see supplementary material, available with the online version of this paper). If possible, sequences of the PCR primers were designed according to previously published data (Faldynova et al., 2003; Matiasovicova et al., 2003; Mikasova et al., 2005; Hermans et al., 2005; Ross \& Heuzenroeder, 2005). If a PCR primer pair was not available, we designed it using GeneCompar software (Applied Maths). After evaluation of the results obtained by individual PCRs, 12 target genes that allowed maximal strain discrimination were selected. To simplify the assay, the PCRs were assembled into four triplex PCRs (Table 2).

Table 2. Primers used in triplex PCRs

\begin{tabular}{|c|c|c|c|}
\hline Triplex PCR & Gene & Primer sequence $\left(5^{\prime}-3^{\prime}\right)$ & Product size $(b p)$ \\
\hline \multirow[t]{6}{*}{ Triplex 1} & allB & F: TTTCGCGACGTTAATGACT & 560 \\
\hline & allB & R: TCAAACATGACGTCCATGC & \\
\hline & SGI1 & F: TTACCGGCGAGTTTACCTC & 438 \\
\hline & SGI1 & R: TCTGCTTGTGTCTTTGGGT & \\
\hline & $r r t T$ & F: CAACGCTCAAGTTCATCTTC & 216 \\
\hline & $r r t T$ & R: AAAGGTGAGCTTGGTGCTC & \\
\hline \multirow[t]{6}{*}{ Triplex 2} & gipA & F: CCTTTGCTAACTTCTTCGC & 450 \\
\hline & gipA & R: GGAGTCGGGCTACCTTTAAG & \\
\hline & $\operatorname{spv} B$ & F: CGGTTATAGAAGAGCTCCTGT & 349 \\
\hline & $\operatorname{spv} B$ & R: CCGGTATACGACTCTGTGATC & \\
\hline & bim2 & F: CCGGTAATGGACTCATTCAG & 206 \\
\hline & bim2 & R: ATCACCATCGAACTGGTTG & \\
\hline \multirow[t]{6}{*}{ Triplex 3} & $g p 36$ & F: GACTAAAGCGGGATACCGTA & 425 \\
\hline & $g p 36$ & R: GGGCTGTCTCTTTCAGCAG & \\
\hline & sopE & F: CCCGTGAAGCTATACTATCG & 348 \\
\hline & $\operatorname{sop} E$ & R: GTTGGAATTGCTGTGGAGT & \\
\hline & artA & F: TCTGGTTATGCAAGTGCTGT & 263 \\
\hline & artA & R: TCTGCACGGATTCTGTATCTA & \\
\hline \multirow[t]{6}{*}{ Triplex 4} & STM2697 & F: ACGAAAAAGCTCAGTAGTGC & 394 \\
\hline & STM2697 & R: TAGTCTATAGCGCCGTTCTC & \\
\hline & hldD & F: CGCAGTAGAGACATGGATGTA & 280 \\
\hline & hldD & R: CTGGCGGTACAGCTTTATG & \\
\hline & gtrA & F: GCGCGTTTCACCTTTAATG & 175 \\
\hline & $g \operatorname{tr} A$ & R: TGGAATAGATAAAGCCGCA & \\
\hline
\end{tabular}


DNA for the PCR was released from a single bacterial colony resuspended in $50 \mu \mathrm{H}_{2} \mathrm{O}$, and incubated for $20 \mathrm{~min}$ in a dry block at $100{ }^{\circ} \mathrm{C}$. PCR was performed with a Multiplex PCR kit from Qiagen, according to the instructions of the manufacturer. After a hot start $\left(15 \mathrm{~min}\right.$ at $\left.95{ }^{\circ} \mathrm{C}\right), 28$ cycles of incubation at $94{ }^{\circ} \mathrm{C}$ for $30 \mathrm{~s}, 60{ }^{\circ} \mathrm{C}$ for $90 \mathrm{~s}$, and $72{ }^{\circ} \mathrm{C}$ for $60 \mathrm{~s}$, were performed. The PCR was terminated by a final extension at $72{ }^{\circ} \mathrm{C}$ for $10 \mathrm{~min}$. The resulting PCR products were resolved by electrophoresis in a $1.5 \%$ agarose gel stained with ethidium bromide, and the amplification products were visualized under UV light.

Results of each PCR were first converted into a binary code: a positive PCR was assigned the value 1 , and a negative PCR was given the value 0 . Since four triplex PCRs were used for strain characterization, each strain was characterized by a raw 12-digit code, such as 101111000 001 . The raw binary code was subsequently transformed into a fourdigit code using the following calculation. The first position in each triplex was given the value 4 , the second position was given the value 2 , and the third position was given the value 1 . Each of these numbers was multiplied by the result of the PCR, and all the values were summed. The first three positions of the above-mentioned triplex PCR should therefore calculate as $(1 \times 4)+(0 \times 2)+(1 \times 1)$, which is equal to 5 . Performing identical calculations with the remaining triplex PCRs gives a PCR code of 5701 in this particular case.

Pulsed-field gel electrophoresis. DNA purification for macrorestriction analysis, restriction enzyme digestion and PFGE were performed essentially as described elsewhere (Hunter et al., 2005). PGFE of XbaI-digested DNA was performed using the CHEF-DRIII system (Bio-Rad) in $0.5 \times$ TBE $(25 \mathrm{mM}$ Tris $/ 25 \mathrm{mM}$ borate $/ 0.5 \mathrm{mM}$ EDTA). After the electrophoresis, the gels were stained with ethidium bromide, and DNA was visualized under UV light.

\section{RESULTS}

\section{Identification of $X b a l$ sites and variable DNA sequences in known $S$. Typhimurium genomes}

When we viewed the distribution of XbaI sites in the genomes of three $S$. Typhimurium strains, we found only a single $\mathrm{XbaI}$ site present at position $4778150 \mathrm{bp}$ of the chromosome of LT2; this site was absent in DT104 strain, and it was associated with a point mutation independent of the prophage sequences. The remaining variation among $S$. Typhimurium LT2, SL1344 and DT104 strains in the predicted restriction fragments generated by $\mathrm{XbaI}$ restriction endonuclease was associated with the presence of prophage sequences or SGI1. This led us to a hypothesis that PCRs specific to these mobile DNA sequences may be of similar discriminatory power, and should correlate with the results of macrorestriction analysis after the digestion with $\mathrm{XbaI}$.

To test this hypothesis, we subjected 58 field strains to PCR using 22 different primer pairs. These PCRs resulted in efficient differentiation between the strains. However, when we analysed the results more carefully, we found that several PCRs did not bring additional value to the system. Typical examples were the $f l j B$ - and oafA-specific PCRs, which yielded positive results in all the strains, and STM908- and STM2705-specific PCRs, which detected Fels1 and Fels2 prophages of the LT2 strain only (for additional information, see supplementary material, avail- able with the online version of this paper). Based on this evaluation, we simplified the PCR typing into 12 PCRs, which were subsequently grouped into four triplex PCRs. The first triplex PCR amplified allB, the left junction of SGI1, and $r r t$. The second triplex PCR was targeted to gipA, spvB and bim2. gipA is encoded by the terminal part of Gifsy $1_{\mathrm{LT} 2}, \operatorname{spv} B$ is encoded by the serovar-specific virulence plasmid, and bim 2 is encoded by the ST64B phage. The third multiplex PCR detected $g p 36$, which is encoded by phage 186 of SL1344, sopE, which is localized on the Fels2 $2_{\text {sopE }}$ phage, and $\operatorname{art} A$, which is localized at the terminal part of the Gifsy $1_{\text {DT104. }}$ The fourth triplex PCR amplified STM2697, which is encoded by both Fels2 and Fels sopE $_{\text {, }}$ hldD, which is characteristic for the cryptic prophage found in the DT104 genome, and gtrA, which is localized on the ST104 phage. The order of the target genes in each triplex PCR was also the order in which the data on positivity or negativity were recorded. The order also corresponded to the size of the PCR products: the first PCR in each triplex resulted in the PCR product with the highest molecular mass, and the last resulted in the smallest PCR product.

Using the multiplex PCR and the whole set of 102 field strains (Fig. 1), 22 multiplex PCR profiles were observed. Using PFGE, 25 profiles were detected. This shows that the system developed is essentially as sensitive as PFGE. Multiplex PCR typing was especially useful in characterization of the DT104 strains and their multidrug-resistant variants. These strains dominated in our collection because of the current epidemiological situation in the Czech Republic, and, although these strains were usually of the same PFGE profile, 11 multiplex PCR types could be identified within the group. Furthermore, among the strains classified as belonging to the phage type DT104, those with a multiplex PCR code starting with 1XXX, 2XXX and 3XXX formed the DT104-like clone. Strains with the PCR code 5XXX, despite being the same phage type as those of the DT104-like clone, did not belong to this clone; this was also confirmed by their different PFGE profiles. Finally, strains of the phage type DT2 were confirmed to form a relatively uniform cluster of strains, by both PCR (Table 3) and PFGE.

When the strains were grouped according to their original host, a specific group of pigeon isolates, characterized by a PCR code 5700, was distinguished. Among the DT104 strains, those that had lost the retron reverse transcriptase locus (code 2313) were found mostly in cattle, and less frequently in pigs and humans, while the retron reversetranscriptase-positive multidrug-resistant strains (strains with PCR code 3313 or 3303) originated mostly from pigs and humans (Tables 3 and 4; see also supplementary material, available with the online version of this paper).

\section{DISCUSSION}

PFGE is considered as a gold standard, not only for $S$. Typhimurium typing, but also for other bacterial species. 


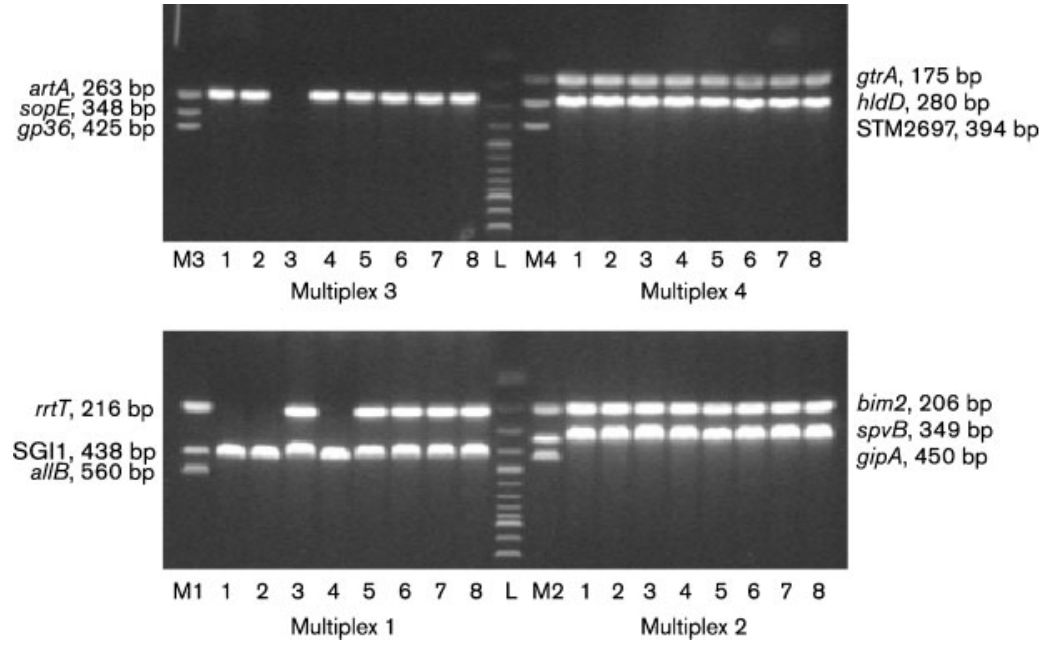

Fig. 1. Multiplex PCR typing of $S$. Typhimurium. Strains in lanes 1-8 were subjected to the multiplex PCR typing. M1-M4, positive multiplex PCR products; L, $100 \mathrm{bp}$ ladder.
In $S$. Typhimurium, although alternative restriction endonucleases can be used, the XbaI restriction endonuclease is the one most frequently used for the digestion of chromosomal DNA. Although XbaI recognizes a $6 \mathrm{bp}$ target sequence, this sequence (TCTAGA) is quite rare in the $S$. Typhimurium genome, and this indicates a negative selection for this sequence during evolution. Fragments of DNA obtained by $S$. Typhimurium in its more recent evolution might therefore contain a higher number of $\mathrm{XbaI}$ target sequences. This situation is different from the NotI restriction endonuclease, for example; this enzyme recognizes an 8 bp target sequence, and the reason for the rare distribution of the target sequences is simply because of the length of recognition sequence. This was a reason why we focused exclusively on $\mathrm{XbaI}$ target sequences in this study. When we analysed genomes of three $S$. Typhimurium strains, we found that almost all of the variation in their XbaI-generated PFGE profiles could be attributed to the presence of prophages. The fact that prophages are major sources of genomic variation in different $S$. Typhimurium strains has been shown by several studies using microarray genomotyping (Porwollik et al., 2004; Reen et al., 2005; Matiasovicova et al., 2007); these studies have indicated that Fels1 and Fels2 prophages are relatively specific for the LT2 strain. Other studies, using conventional PCR, have found that sopE, encoded by a Fels-like prophage, is quite uncommon to S. Typhimurium (Mirold et al., 1999). This information has led to studies that have used the detection of prophage sequences for strain characterization (Mikasova et al., 2005; Hermans et al., 2005; Ross \& Heuzenroeder, 2005); however, the approach in those studies was rather arbitrary. In this study, we used accumulated genomic data, either from genome sequencing (McClelland et al., 2001) or from microarray genomotyping (Porwollik et al., 2004; Reen et al., 2005; Matiasovicova et al., 2007), for rational design of a PCRtyping system based on the presence or absence of mobile DNA sequences. Multiplexing of the specific PCR further

Table 3. Distribution of phage types and multiplex PCR types among S. Typhimurium field strains analysed in this study

\begin{tabular}{|lrl|}
\hline Phage type & No. of strains & \multicolumn{1}{c|}{ Multiplex PCR type $^{*}$} \\
\hline DT1 & 3 & $5101(1), 5700(1), 1200(1)$ \\
DT2 & 10 & $5700(8), 5701(1), 5724(1)$ \\
DT12 & 1 & $3313(1)$ \\
DT14 & 1 & $5565(1)$ \\
DT104 & 62 & $1303(1), 1313(14), 2313(6), 2333(1), 3303(6), 3313(28), 3337(1), 5101(1), 5700(1), 5724(2), 5765(1)$ \\
DT114 & 1 & $5724(1)$ \\
DT120 & 3 & $3313(2), 2313(1)$ \\
DT166 & 1 & $1310(1)$ \\
DT193 & 1 & $5524(1)$ \\
DT195 & 1 & $5700(1)$ \\
U & 2 & $5640(1), 1310(1)$ \\
RDNC & 3 & $5524(1), 5525(1), 5404(1)$ \\
Not known & 13 & $1313(3), 3313(2), 5765(2), 5501(2), 5604(1), 5764(1), 2313(1), 5724(1)$ \\
\hline
\end{tabular}

*Values in parentheses indicate the number of times that a particular multiplex PCR type was detected. 
Table 4. Distribution of different multiplex PCR types among $S$. Typhimurium field strains originating from various hosts

\begin{tabular}{|lcl|}
\hline Host & No. of strains & \multicolumn{1}{c|}{ Multiplex PCR type $^{*}$} \\
\hline Human & 23 & $3313(11), 1313(5), 3303(3), 2313(1), 5640(1), 5700(1), 5724(1)$ \\
Pig & 21 & $3313(13), 3303(2), 2313(2), 5724(2), 1313(1), 3337(1)$ \\
Cattle & 24 & $1313(6), 2313(5), 3313(4), 5524(2), 5765(2), 1303(1), 5101(1), 5525(1), 5565(1), 5724(1)$ \\
Pigeon & 13 & $5700(8), 3313(2), 1310(1), 5701(1), 5724(1)$ \\
Poultry & 10 & $1313(3), 5501(2), 3313(1), 2333(1), 5101(1), 5700(1), 5765(1)$ \\
Environment & 2 & $3313(1), 1313(1)$ \\
Cat & 2 & $3303(1), 3313(1)$ \\
Duck & 1 & $5700(1)$ \\
Food & 1 & $1313(1)$ \\
Pheasant & 1 & $5404(1)$ \\
Parrot & 1 & $1310(1)$ \\
Wild boar & 1 & $1200(1)$ \\
Not known & 2 & $5604(1), 5764(1)$ \\
\hline
\end{tabular}

*Values in parentheses indicate the number of times that a particular multiplex PCR type was detected.

simplified the protocol, and made it user friendly. When compared with the PFGE, the system was essentially of the same discriminatory power. However, unlike PFGE, the multiplex PCR typing system can be easily adopted by any laboratory capable of running PCR, thus allowing simple data comparison. A further advantage of the proposed system is that it remains open for the introduction of additional PCRs when new sequence data become available. In such cases, a new target sequence can be added to the current sequences, provided that the order of the PCRs is not changed.

\section{ACKNOWLEDGEMENTS}

This work has been supported by projects 1 B40019 and MZE0002716201 of the Czech Ministry of Agriculture. The authors wish to acknowledge the excellent technical assistance of Michaela Dekanova.

\section{REFERENCES}

Anderson, E. S., Ward, L. R., Saxe, M. J. \& de Sa, J. D. (1977). Bacteriophage-typing designations of Salmonella typhimurium. J Hyg (Lond) 78, 297-300.

Faldynova, M., Pravcova, M., Sisak, F., Havlickova, H., Kolackova, I., Cizek, A., Karpiskova, R. \& Rychlik, I. (2003). Evolution of antibiotic resistance in Salmonella enterica serovar Typhimurium strains isolated in the Czech Republic between 1984 and 2002. Antimicrob Agents Chemother 47, 2002-2005.

Galanis, E., Lo Fo Wong, D. M., Patrick, M. E., Binsztein, N., Cieslik, A., Chalermchikit, T., Aidara-Kane, A., Ellis, A., Angulo, F. J. \& Wegener, H. C. (2006). Web-based surveillance and global Salmonella distribution, 2000-2002. Emerg Infect Dis 12, 381-388.

Hermans, A. P., Abee, T., Zwietering, M. H. \& Aarts, H. J. (2005). Identification of novel Salmonella enterica serovar Typhimurium DT104-specific prophage and nonprophage chromosomal sequences among serovar Typhimurium isolates by genomic subtractive hybridization. Appl Environ Microbiol 71, 4979-4985.
Hradecka, H., Kolackova, l., Karpiskova, R. \& Rychlik, I. (2006). An outbreak of human salmonellosis caused by ampicillin-resistant Salmonella enterica serovar Enteritidis PT13 in the Czech Republic. Epidemiol Infect 134, 737-740.

Hunter, S. B., Vauterin, P., Lambert-Fair, M. A., Van Duyne, M. S., Kubota, K., Graves, L., Wrigley, D., Barrett, T. \& Ribot, E. (2005). Establishment of a universal size standard strain for use with the PulseNet standardized pulsed-field gel electrophoresis protocols: converting the national databases to the new size standard. J Clin Microbiol 43, 1045-1050.

Matiasovicova, J., Faldynova, M., Pravcova, M., Karpiskova, R., Kolackova, I., Damborsky, J. \& Rychlik, I. (2003). Retron reverse transcriptase $r r t T$ is ubiquitous in strains of Salmonella enterica serovar Typhimurium. FEMS Microbiol Lett 223, 281-286.

Matiasovicova, J., Adams, P., Barrow, P. A., Hradecka, H., Malcova, M., Karpiskova, R., Budinska, E., Pilousova, L. \& Rychlik, I. (2007). Identification of putative ancestors of the multidrug-resistant Salmonella enterica serovar Typhimurium DT104 clone harboring the Salmonella genomic island 1. Arch Microbiol 187, 415-424.

McClelland, M., Sanderson, K. E., Spieth, J., Clifton, S. W., Latreille, P., Courtney, L., Porwollik, S., Ali, J., Dante, M. \& other authors (2001). Complete genome sequence of Salmonella enterica serovar Typhimurium LT2. Nature 413, 852-856.

Mikasova, E., Drahovska, H., Szemes, T., Kuchta, T., Karpiskova, R., Sasik, M. \& Turna, J. (2005). Characterization of Salmonella enterica serovar Typhimurium strains of veterinary origin by molecular typing methods. Vet Microbiol 109, 113-120.

Mirold, S., Rabsch, W., Rohde, M., Stender, S., Tschape, H., Russmann, H., Igwe, E. \& Hardt, W. D. (1999). Isolation of a temperate bacteriophage encoding the type III effector protein SopE from an epidemic Salmonella typhimurium strain. Proc Natl Acad Sci U S A 96, 9845-9850.

Porwollik, S., Boyd, E. F., Choy, C., Cheng, P., Florea, L., Proctor, E. \& McClelland, M. (2004). Characterization of Salmonella enterica subspecies I genovars by use of microarrays. J Bacteriol 186, 5883-5898.

Reen, F. J., Boyd, E. F., Porwollik, S., Murphy, B. P., Gilroy, D., Fanning, S. \& McClelland, M. (2005). Genomic comparisons of Salmonella enterica serovar Dublin, Agona, and Typhimurium strains recently isolated from milk filters and bovine samples from Ireland, using a Salmonella microarray. Appl Environ Microbiol 71, 1616-1625. 
Ross, I. L. \& Heuzenroeder, M. W. (2005). Discrimination within phenotypically closely related definitive types of Salmonella enterica serovar Typhimurium by the multiple amplification of phage locus typing technique. J Clin Microbiol 43, 1604-1611.

Van Immerseel, F., Pasmans, F., De Buck, J., Rychlik, l., Hradecka, H., Collard, J. M., Wildemauwe, C., Heyndrickx, M., Ducatelle, R. \&
Haesebrouck, F. (2004). Cats as a risk for transmission of antimicrobial drug-resistant Salmonella. Emerg Infect Dis 10, 2169-2174.

Edited by: D. W. Ussery 